\title{
Evaluating Multimodal Driver Displays of Varying Urgency for Drivers on the Autistic Spectrum
}

\author{
Leeseul Shim \\ School of Psychology \\ University of Glasgow \\ Glasgow, G12 8QB, UK \\ L.Shim.1@research.gla.ac.uk \\ Paula Regener \\ School of Psychology \\ University of Glasgow \\ Glasgow, G12 8QB, UK \\ P.Regener.1@research.gla.ac.uk
}

\author{
Peipei Liu \\ School of Psychology \\ University of Glasgow \\ Glasgow, G12 8QB, UK \\ P.Liu.2@research.gla.ac.uk \\ Stephen Brewster \\ School of Computing Science \\ University of Glasgow \\ Glasgow, G12 8QQ, UK \\ Stephen.Brewster@glasgow.ac.uk
}

\author{
Ioannis Politis \\ School of Computing Science \\ University of Glasgow \\ Glasgow, G12 8QQ, UK \\ I.Politis.1@research.gla.ac.uk \\ Frank Pollick \\ School of Psychology \\ University of Glasgow \\ Glasgow, G12 8QB, UK \\ Frank.Pollick@glasgow.ac.uk
}

\begin{abstract}
This paper examines the response of 10 typical and 10 autism spectrum participants in their reaction to a set of uniand multisensory warning signals designed to indicate different levels of urgency. The warnings were composed of auditory, visual and tactile signals that were presented alone or in combination. Two experiments were conducted, a first that examined perceived urgency and annoyance with the warnings and a second that used a driving simulator scenario to explore recognition of the level of urgency and the speed of response. Results of Experiment 1 showed that there was no difference between groups in the perceived urgency of the warning signals, though the autism spectrum group reported less annoyance with the signals. Results of Experiment 2 showed that while both groups showed high accuracy in correctly reporting urgency level, the autism spectrum group performed better. Moreover, the fastest overall reaction times obtained were by the autism spectrum group when the warning included a visual component, with vision alone producing the quickest response. These results provide novel empirical insights on behaviour of drivers with autism when exposed to multimodal driver displays. They also highlight how consideration of characteristics of individual differences can contribute to the design of effective warning signals.
\end{abstract}

\section{Author Keywords}

Autism; multimodal interaction; warnings; audio; visual; tactile; perceived urgency; perceived annoyance;

\footnotetext{
Permission to make digital or hard copies of all or part of this work for personal or classroom use is granted without fee provided that copies are not made or distributed for profit or commercial advantage and that copies bear this notice and the full citation on the first page. Copyrights for components of this work owned by others than ACM must be honored. Abstracting with credit is permitted. To copy otherwise, or republish, to post on servers or to redistribute to lists, requires prior specific permission and/or a fee. Request permissions from Permissions@acm.org. AutomotiveUI '15, September 01 - 03, 2015, Nottingham, UK (C) 2015 ACM. ISBN 978-1-4503-3736-6/15/09...\$15.00 DOI: http://dx.doi.org/10.1145/2799250.2799261.
}

recognition time; simulator.

\section{ACM Classification Keywords}

H.5.2 [Information interfaces and presentation]: User Interfaces. - Auditory (non-speech) feedback; Haptic I/O; Voice I/O, J.4 [Social and behavioral sciences]: Psychology.

\section{INTRODUCTION}

Recent studies have examined the design of warning signals and how to effectively combine different sensory modalities to create multisensory warning signals [4,13,17,21,22,27]. This evidence typically points towards improvements, or at the least no decrements with multisensory warning signals, a result that is consistent with psychological theories that suggest how multiple senses can be combined optimally [10]. However, these studies on multisensory warning signals have been performed on typical populations and little is known how different subpopulations might react to these multisensory warning signals. Individuals on the autism spectrum are an interesting subpopulation to study as differences in sensory and multisensory processing have been reported. With a general prevalence in the USA of 1 in 68 children of which approximately half have average to above average intelligence [5] there are potential implications for a large number of drivers.

Autism Spectrum Disorders (ASD), represent a class of highly ubiquitous developmental disabilities that have been characterised by deficits in communication and social reciprocity, and by the presence of restricted and/or repetitive behaviours [1]. Individuals with ASD also demonstrate alterations in sensory processing within individual modalities (e.g., vision, hearing, touch, and proprioception) and uncommon responses to sensory stimuli across multiple sensory domains [15]. It is important to note that abnormal sensory processing does not necessarily mean worse performance, as there is evidence of enhanced perceptual functioning in autism [3,19]. Consistent with evidence from perception of single senses, recent results show that multisensory perception differs 
between Typically Developed (TD) and ASD groups $[2,11,16,18,29]$. These differences in perception can lead to difficulties in the integration of multiple sensory signals in everyday situations.

It is thus a relevant question whether the gains reported from multisensory warning signals in typical individuals will apply to individuals on the autism spectrum. Given the difficulties reported in previous research conducted under laboratory conditions it would seem likely that differences would be found between ASD and TD groups and that these basic differences might be accentuated by the sensory and cognitive load of driving. The limited research investigating driving in autism has found differences between ASD and TD groups that is consistent with reduced effectiveness in driving. However, some of this research is based on self-report and report of parents and caregivers. For example, surveys have shown that $63 \%$ of teenagers on the autism spectrum plan to obtain their driving license [14], even though their parents believe this is a challenging goal and one that the parents also believe that they might have substantial difficulty at performing safely [7]. A study by [8] used self-reports from a standardized driver behaviour questionnaire to examine differences between ASD and TD adults (mean age 33). Results showed that ASD drivers acquired licenses significantly later, drove significantly fewer days per week, and reported more traffic violations than non-ASD drivers. They were also more likely to report themselves as "poor drivers". However, their assessment as poor drivers did not relate to reports of poor driving behaviour, raising the issue of how differences in self-appraisal might contribute to differences found between ASD and TD groups.

Perceptual experiments have found differences between ASD and TD groups. Sheppard and colleagues [28] examined detection of driving hazards and found that individuals on the autism spectrum were less accurate at reporting hazards arising from social activity and overall had slower reactions times in reporting both social and nonsocial events. Using a driving simulator, in a small study of 7 teenagers on the autism spectrum, it was reported that the ASD teenagers compared to TD were involved in a greater number of accidents such as off-road crashes, collisions and centre-line crossings [6]. Another simulator study by Reimer and colleagues [24] used a driving simulator to explore physiological (Heart Rate, Skin Conductance) and eye movement responses to simulated driving in young drivers (18-24) who were either on the autism spectrum or in a matched group of typical drivers. They found that while the typical drivers showed signs of increased arousal to cognitive load, the ASD group did not. However, the ASD group did show greater horizontal deviation from forward and towards oncoming traffic in the opposing lane with cognitive load. Additionally, across all experimental conditions the ASD group placed their gaze much higher in the visual field; a result consistent with attending to objects and events further ahead in the distance. These eye- tracking results suggest ASD drivers place less attention on the vehicle directly in front.

To summarise, different modes of sensory information (touch, sight, sound) can be used to warn a driver and multisensory warnings incorporating two or more modalities show promise in their effectiveness. However, most studies exploring the effectiveness of warning signals have used typical participants and thus little is known about how effective the different sensory modalities and their combinations are in different subpopulations. In this research we explore one such population, those with a high degree of autistic traits as measured by their Autism Quotient score [30]. We stress that this is not equivalent to a formal diagnosis of autism. This subpopulation was chosen since not only does early evidence indicate general issues with driving in autism but specific claims about multisensory perception in autism suggest that these individuals integrate across the senses in ways different from the typical population. In two experiments we contrast responses of typically developed individuals to those on the autism spectrum using a collection of uni and multisensory warning signals that have been designed to indicate different levels of urgency [22]. In the first experiment we explore perceived urgency and annoyance with the warnings, while in the second experiment we investigate recognition accuracy and response times.

\section{WARNING DESIGN}

The set of warnings used in this study were similar to [22]. Three Levels of Designed Urgency (LDU) were created to signify various situations on the road. All unimodal, bimodal and trimodal combinations of audio, visual and tactile modalities were used in the warnings: Audio (A), Visual (V), Tactile (T), Audio + Visual (AV), Audio + Tactile (AT), Tactile + Visual (TV), Audio + Tactile + Visual (ATV). This resulted in 21 different signals: 7 modalities $\times 3$ levels of designed urgency.

Pure tones, colours or vibrations were used in the warnings as pulses. Depending on the level of urgency, pulse rate varied, increasing as signals increased in urgency. Warnings of the same urgency level had the same pulse rate irrespective of modality. In addition, peak values of intensity for each LDU were adjusted in line with [23] since this has decreased annoyance ratings in the past. The values of intensity used were identical to [23] in each respective urgency level. Thus, 8 pulses with $0.1 \mathrm{sec}$ single pulse duration, $0.1 \mathrm{sec}$ interpulse interval and a peak of $-1.9 \mathrm{dBFS}$ were used for $\mathrm{L}_{\mathrm{H}}, 5$ pulses with $0.17 \mathrm{sec}$ single pulse duration, $0.17 \mathrm{sec}$ interpulse interval and a peak of -11.1 $d B F S$ for $\mathrm{L}_{\mathrm{M}}$ and 2 pulses with $0.5 \mathrm{sec}$ single pulse duration, $0.5 \mathrm{sec}$ interpulse interval and a peak of -16.5 $d B F S$ for $\mathrm{L}_{\mathrm{L}}$. All warnings lasted $1.5 \mathrm{sec}$ each.

As in [22], auditory warnings were additionally varied in base frequency $\left(1000 \mathrm{~Hz}\right.$ for $\mathrm{L}_{\mathrm{H}}, 700 \mathrm{~Hz}$ for $\mathrm{L}_{\mathrm{M}}$ and $400 \mathrm{~Hz}$ for $\left.\mathrm{L}_{\mathrm{L}}\right)$. Visual warnings were varied in colour, Red $(R G B(255,0,0))$ for $\mathrm{L}_{\mathrm{H}}$, Orange $(R G B(255,127,0))$ for $\mathrm{L}_{\mathrm{M}}$ 
and Yellow $(R G B(255,255,0))$ for $\mathrm{L}_{\mathrm{L}}$. A C2 Tactor from Engineering Acoustics was used for the tactile stimuli, which had a constant frequency of $250 \mathrm{~Hz}$. Simultaneous delivery of unimodal signals was used for multimodal ones, to create a synchronous effect.

To evaluate the warnings created, two experiments presented in [22] were replicated, looking into perceived urgency and annoyance of the signals as well as recognition time and accuracy, when comparing responses of control participants versus participants on the spectrum of autism. We compared a TD group, forming the Control group for this study, with an ASD group.

\section{EXPERIMENT 1}

The first experiment investigated the subjective responses in terms of perceived urgency and perceived annoyance. In line with [22], it was hypothesized that the different modalities and levels of urgency designed in the warnings would influence the ratings of urgency and annoyance. Further, any differences in subjective responses between control and ASD group would be identified.

\section{Design}

A $7 \times 3 \times 2$ mixed design was followed for this experiment, with Modality, LDU and Group as the independent variables and Perceived Urgency (PU) and Perceived Annoyance (PA) as the dependent ones. Modality and LDU were within subjects variables and Group was a between subjects variable. Modality had 7 levels: A, T, V, AT, AV, TV, ATV. LDU had 3 levels: $\mathrm{L}_{\mathrm{H}}, \mathrm{L}_{\mathrm{M}}$ and $\mathrm{L}_{\mathrm{L}}$. Group had 2 levels: ASD and Control (TD).

Twenty male participants took part in this experiment, ten in control group and ten in ASD group. The criterion for grouping participants in ASD group was a self-reported diagnosis of Autism. Further, participants were grouped in control group when there was no such diagnosis. Both the ASD and Control groups were measured on the Autism Quotient [30], which rate autistic traits on a scale of 1 to 50 with high scores indicating more autistic traits. A criterion of a score lower than 26 was used to enter the Control group and average scores of 13.6 (SD 5.03) and 40.3 (SD 6.22) were obtained for the Control and ASD groups respectively. All participants reported normal or corrected to normal vision and normal hearing. All except one in ASD group held a valid driving licence and all except one in ASD group were right handed. Ages between groups were matched, as an independent samples $t$-test showed no significant difference of age between groups. Control group participants' ages varied from 20 to 47 years $(M=34.50$, $S D=10.71)$ and their driving experience from 2 to 27 years $(M=14.70, S D=10.13)$. ASD group participants' were 22 - 52 years $(M=31.90, S D=10.30)$ and their driving experience from 2 to 31 years $(M=9.60, S D=10.82)$.

\section{Procedure}

Procedure of the experiment was similar to Experiment 1 in [22] and was identical for both control and ASD groups.
Participants sat in front of a 27- inch computer screen with a computer running the driving simulator software. A threelane road in a rural area and a front car maintaining a steady speed of about $60 \mathrm{mph}$ was depicted. A set of headphones (Beyerdynamic DT) was used for sound and the C2 Tactor for vibration, attached on a wrist band on the left hand, as in [23] (see Figure 1.c). Visuals were delivered through coloured circles that flashed in the top centre of the screen, sized $400 \times 400$ pixels $(11 \times 11 \mathrm{~cm})$. The circles did not obstruct the view of the road and were simulating a HeadUp Display (HUD). A mouse was used to submit ratings.

Participants were welcomed and provided an introduction to the experiment. Car sound was heard throughout the experiment to cover the Tactor noise. For familiarization with the cues, all 21 signals were played once to the participants, always in the following order: $\mathrm{A} \rightarrow \mathrm{V} \rightarrow \mathrm{T} \rightarrow$ $\mathrm{AV} \rightarrow \mathrm{AT} \rightarrow \mathrm{TV} \rightarrow \mathrm{ATV}$ for $\mathrm{L}_{\mathrm{H}}$, then for $\mathrm{L}_{\mathrm{M}}$ and then for $\mathrm{L}_{\mathrm{L}}$. If needed, sound and vibration were adjusted for comfort. No information on LDU was given. Next, the warning signals were played in a random order and with a random interval of any integral value between (and including) $8-20 \mathrm{sec}$, as in [22]. Each stimulus was played 3 times. This resulted in a total of 63 trials. For each stimulus, participants were asked to rate perceived urgency and annoyance on a scale of 0 to 100 ( 0 for lowest, 100 for highest), in line with [22]. Participants were looking at the driving simulator with the car throughout this task. After rating the stimuli participants' perceived workload of this task was assessed using the Driving Activity Load Index (DALI) [20]. The experiment lasted about 30 minutes and participants were then prepared for Experiment 2 in the same session. See Figure 1.a for the setup of Experiment 1.
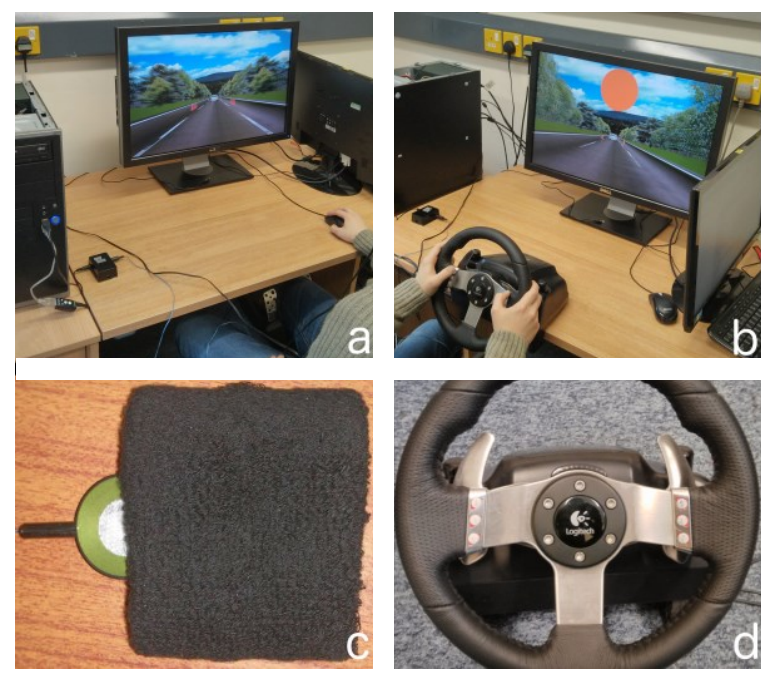

Figure 1: The setup of Experiments 1 (a) and 2 (b), the wristband with the Tactor (c) and the steering wheel used in Experiment 2, with the response buttons (d). 


\section{Results}

\section{Perceived Urgency}

Data for PU were analysed using a three-way mixed ANOVA, with Modality and LDU as within subjects and Group as a between subjects factor. Due to sphericity violations, degrees of freedom were corrected using Greenhouse-Geisser estimates. There was a significant main effect of Modality $(F(4.52,262.41)=56.72, p<$ $0.001)$. Contrasts revealed that modalities were rated for $\mathrm{PU}$ in the following order: A lower than $\mathrm{V}(F(1,58)=7.98, r=$ $0.35, p<0.01)$, $\mathrm{V}$ lower than $\mathrm{T}(F(1,58)=7.08, r=0.33, p$ $<0.05)$, T lower than AV and AT $(F(1,58)=5.31, r=0.29$, $p<0.05)$, AV and AT lower than TV $(F(1,58)=4.18, r=$ $0.26, p<0.05)$ and TV lower than $\operatorname{ATV}(F(1,58)=20.90, r$ $=0.51, p<0.001)$. There was a significant main effect of LDU $(F(1.30,75.32)=245.80, p<0.001)$. Contrasts revealed that $\mathrm{L}_{\mathrm{L}}$ was rated lower than $\mathrm{L}_{\mathrm{M}}(F(1,58)=112.80$, $r=0.81, p<0.001)$ and $\mathrm{L}_{\mathrm{M}}$ lower than $\mathrm{L}_{\mathrm{H}}(F(1,58)=$ 370.47, $r=0.93, p<0.001)$. There was a significant interaction between Modality and LDU $(F(9.12,528.93)=$ 5.98, $p<0.001)$. Contrasts revealed that although PU was higher in $\mathrm{L}_{\mathrm{L}}$ for $\mathrm{T}$ compared to $\mathrm{V}(F(1,58)=8.94, r=0.37$, $p<0.01)$ and $\operatorname{AV}(F(1,58)=4.04, r=0.25, p<0.05)$, it was lower compared to $\mathrm{AV}$ in $\mathrm{L}_{\mathrm{H}}(F(1,58)=12.00, r=$ $0.41, p<0.01)$. Also, that in $\mathrm{L}_{\mathrm{L}}$ there was no difference in PU between TV and ATV $(F(1,58)=5.34, r=0.29, p<$ $0.05)$. There was no significant main effect of Group. Figure 2.a shows mean perceived urgency values across groups. Table 1 shows mean values and confidence intervals for all measures in both experiments.

\section{Perceived Annoyance}

Data for PA were analysed using a three-way mixed ANOVA, with Modality and LDU as within subjects and Group as a between subjects factor. Due to sphericity violations, degrees of freedom were corrected using Greenhouse-Geisser estimates. There was a significant main effect of Modality $(F(2.49,144.12)=24.61, p<$ $0.001)$. Contrasts revealed that modalities were rated for PA in the following order: $\mathrm{V}$ and $\mathrm{A}$ lower than $\mathrm{AV}$ and $\mathrm{T}$ $(F(1,58)=11.32, r=0.40, p<0.01)$, and $\mathrm{AV}$ and $\mathrm{T}$ lower than AT, TV and $\operatorname{ATV}(F(1,58)=17.29, r=0.48, p<$ $0.001)$. There was a significant main effect of LDU $(F(1.34,77.69)=29.44, p<0.001)$. Contrasts revealed that $\mathrm{L}_{\mathrm{L}}$ was rated lower than $\mathrm{L}_{\mathrm{M}}(F(1,58)=16.15, r=0.47, p<$ $0.001)$ and $\mathrm{L}_{\mathrm{M}}$ lower than $\mathrm{L}_{\mathrm{H}}(F(1,58)=30.98, r=0.59, p<$ 0.001). There was a significant interaction between Modality and LDU $(F(8.99,521.14)=2.07, p<0.05)$. Contrasts revealed that ratings of PA for ATV were higher in $\mathrm{L}_{\mathrm{H}}$ and did not differ in $\mathrm{L}_{\mathrm{M}}$ and $\mathrm{L}_{\mathrm{L}}(F(1,58)=4.14, r=$ $0.26, p<0.05)$. Finally, there was a significant main effect of Group, revealing that PA ratings were lower for ASD group $(F(1,58)=13.22, r=0.43, p<0.01)$. See Figure 2.a for mean perceived annoyance values across groups.
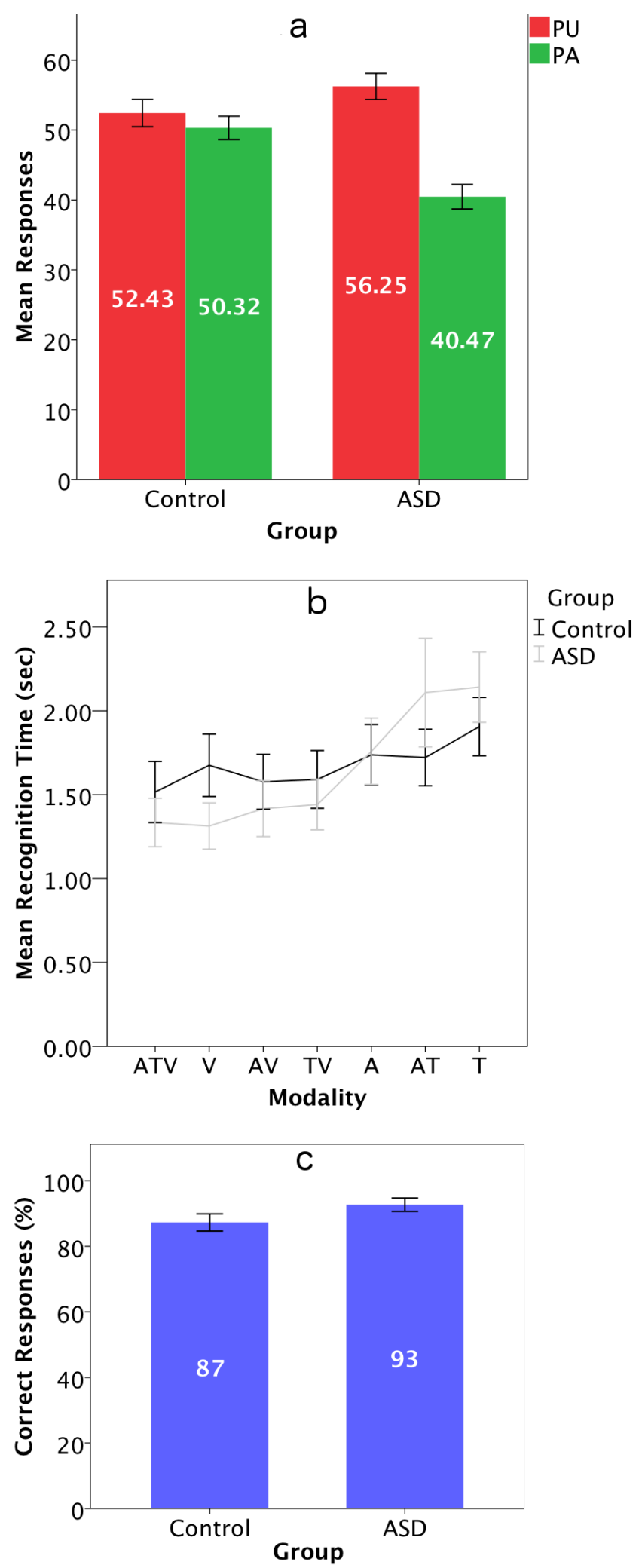

Figure 2: (a) Perceived Urgency (PU) and Perceived Annoyance (PA) across Groups. The scale for PU and PA was from 0 to 100. (b) Interaction between Modality and Group for Recognition Time. (c) Recognition Accuracy across Groups. Error bars indicate $\mathbf{9 5 \%}$ confidence intervals. 


\begin{tabular}{|c|c|c|c|c|c|}
\hline Measure & & Factor & Mean & Lower & Upper \\
\hline \multirow{12}{*}{$\begin{array}{c}\text { PU } \\
(\mathbf{0 - 1 0 0 )})\end{array}$} & \multirow{7}{*}{ 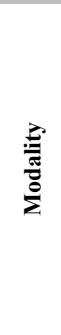 } & A & 41.87 & 38.49 & 45.24 \\
\hline & & $\mathrm{V}$ & 46.61 & 43.68 & 49.54 \\
\hline & & $\mathrm{T}$ & 51.51 & 47.73 & 55.28 \\
\hline & & AV & 55.76 & 52.46 & 59.07 \\
\hline & & $\mathrm{AT}$ & 58.21 & 54.43 & 61.99 \\
\hline & & $\mathrm{TV}$ & 61.10 & 58.12 & 64.08 \\
\hline & & ATV & 65.33 & 62.69 & 67.97 \\
\hline & \multirow{3}{*}{ 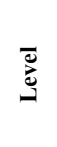 } & $\mathrm{L}_{\mathrm{H}}$ & 73.88 & 70.32 & 77.45 \\
\hline & & $\mathrm{L}_{\mathrm{M}}$ & 53.94 & 50.66 & 57.21 \\
\hline & & $\mathrm{L}_{\mathrm{L}}$ & 35.20 & 32.25 & 38.15 \\
\hline & \multirow{2}{*}{ 言 } & Control & 52.43 & 48.79 & 56.08 \\
\hline & & ASD & 56.25 & 52.60 & 59.90 \\
\hline \multirow{12}{*}{$\begin{array}{c}\text { PA } \\
(0-100)\end{array}$} & \multirow{7}{*}{ 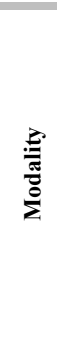 } & V & 33.72 & 28.79 & 38.66 \\
\hline & & A & 36.27 & 32.52 & 40.03 \\
\hline & & $\mathrm{AV}$ & 42.41 & 38.83 & 46.00 \\
\hline & & $\mathrm{T}$ & 46.49 & 42.32 & 50.67 \\
\hline & & $\mathrm{AT}$ & 52.03 & 48.37 & 55.70 \\
\hline & & $\mathrm{TV}$ & 52.07 & 47.80 & 56.34 \\
\hline & & ATV & 54.75 & 50.62 & 58.88 \\
\hline & \multirow{3}{*}{ 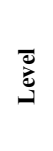 } & $\mathrm{L}_{\mathrm{H}}$ & 52.19 & 48.93 & 55.45 \\
\hline & & $\mathrm{L}_{\mathrm{M}}$ & 44.91 & 42.18 & 47.64 \\
\hline & & $\mathrm{L}_{\mathrm{L}}$ & 39.08 & 35.12 & 43.04 \\
\hline & \multirow{2}{*}{ 言 } & Control & 50.32 & 46.49 & 54.15 \\
\hline & & ASD & 40.47 & 36.64 & 44.30 \\
\hline \multirow{12}{*}{$\begin{array}{l}\text { RT } \\
\text { (sec) }\end{array}$} & \multirow{7}{*}{ 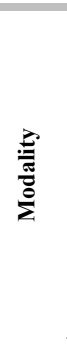 } & ATV & 1.42 & 1.26 & 1.59 \\
\hline & & $\mathrm{V}$ & 1.47 & 1.30 & 1.64 \\
\hline & & AV & 1.48 & 1.31 & 1.64 \\
\hline & & TV & 1.50 & 1.34 & 1.66 \\
\hline & & $\mathrm{A}$ & 1.74 & 1.57 & 1.91 \\
\hline & & AT & 1.87 & 1.67 & 2.08 \\
\hline & & $\mathrm{T}$ & 2.01 & 1.84 & 2.18 \\
\hline & \multirow{3}{*}{$\bar{\Xi}$} & $\mathrm{L}_{\mathrm{H}}$ & 1.32 & 1.19 & 1.46 \\
\hline & & $\mathrm{L}_{\mathrm{M}}$ & 1.78 & 1.62 & 1.94 \\
\hline & & $\mathrm{L}_{\mathrm{L}}$ & 1.82 & 1.64 & 2.00 \\
\hline & \multirow{2}{*}{ 言 } & Control & 1.68 & 1.47 & 1.88 \\
\hline & & ASD & 1.61 & 1.40 & 1.82 \\
\hline
\end{tabular}

Table 1: The mean values, upper $95 \%$ confidence intervals (Upper) and lower 95\% confidence intervals (Lower) of Perceived Urgency (PU), Perceived Annoyance (PA) and Recognition Time (RT) in the two experiments.

Experiment 1 confirmed the effects found in [22], and showed that all participants recognised the designed urgency in the warnings. Warnings of higher urgency were perceived as such, with a similar increase in perceived annoyance with lower effects. More modalities increased ratings, while the ASD group was found to provide lower ratings of annoyance overall. To assess objective responses to the warnings and any differences between groups in a recognition task, Experiment 2 was conducted immediately after Experiment 1.

\section{EXPERIMENT 2}

For the second experiment, the same warnings were evaluated for recognition time and accuracy. As in [22] it was hypothesized that the designed urgency and modality of warnings would influence their recognition time. Further, any differences between control and ASD groups would also be identified for this task.

\section{Design}

As in Experiment 1, a $7 \times 3 \times 2$ mixed design was followed for this experiment, with Modality, LDU and Group as the independent variables and Recognition Time (RT) and Recognition Accuracy (RA) as the dependent ones. Modality and LDU were within subjects variables and Group was a between subjects variable. Participants were identical to Experiment 1. Equipment was identical, with the addition of a Logitech G27 gaming wheel to control the simulated vehicle and provide responses. The simulator logged inputs at a frequency of $50 \mathrm{~Hz}$.

\section{Procedure}

Procedure of the experiment was similar to Experiment 2 in [22] and was identical for both control and ASD groups. Before beginning the new experimental task, all 21 signals were played once to the participants. A label with the text "Level H (HIGH): Warnings of HIGH urgency e.g. Impending Collision" was presented followed by all cues of $\mathrm{L}_{\mathrm{H}},(\mathrm{A} \rightarrow \mathrm{V} \rightarrow \mathrm{T} \rightarrow \mathrm{AV} \rightarrow \mathrm{AT} \rightarrow \mathrm{TV} \rightarrow \mathrm{ATV})$. This was followed by a label reading "Level $M$ (MEDIUM): Warnings of MEDIUM urgency e.g. Low Fuel" and the cues of $L_{M}$ in the same order. Finally, a label with the text "Level L (LOW): Warnings of LOW urgency e.g. Incoming Message" was shown, followed by the cues of $\mathrm{L}_{\mathrm{L}}$. For the main experiment, the warnings were played to the participants in a random order and with a random interval of any integral value between (and including) $8-20 \mathrm{sec}$, as in Experiment 1. Each stimulus was played 3 times, resulting in 63 trials. Participants were asked to identify the level of urgency of each stimulus by pressing one of three labelled buttons on the steering wheel as quickly as possible. Buttons were labelled with letters $(\mathrm{H}, \mathrm{M}$ or L) according to the urgency levels - topmost for $\mathrm{L}_{\mathrm{H}}$, middle for $\mathrm{L}_{\mathrm{M}}$, bottom for $\mathrm{L}_{\mathrm{L}}$ (see Figure 1.d). While performing the above task, participants were steering a simulated vehicle maintaining a speed of about $60 \mathrm{mph}$ and were instructed to maintain a central position in the lane. The accelerator and brake pedals were not used. Finally, they were asked to fill in the DALI questionnaire to assess their perceived workload for this task. The experiment lasted about 20 minutes and participants received $£ 6$ for participating to both experiments. See Figure 1.b for the setup of Experiment 2. 


\section{Results}

\section{Recognition Time}

Data for RT were analysed using a three-way mixed ANOVA, with Modality and LDU as within subjects and Group as a between subjects factor. Due to sphericity violations, degrees of freedom were corrected using Greenhouse-Geisser estimates. There was a significant main effect of Modality $(F(4.12,235.00)=24.42, p<$ 0.001). Contrasts revealed that ATV, V, AV and TV created quicker responses compared to A, AT and T $(F(1,57)=12.30, r=0.42, p<0.01)$. There was a significant main effect of $\operatorname{LDU}(F(1.76,100.34)=57.12, p$ $<0.001)$. Contrasts revealed that $\mathrm{L}_{\mathrm{H}}$ warnings created quicker responses compared to $\mathrm{L}_{\mathrm{M}}$ and $\mathrm{L}_{\mathrm{L}}(F(1,57)=$ $120.03, r=0.82, p<0.001)$. There was no significant main effect of Group, but a significant interaction between Group and Modality $(F(4.12,235.00)=7.23, p<0.001)$, and Group and LDU $(F(1.76,100.34)=6.93, p<0.01)$. Contrasts revealed that ASD group had quicker responses in modality $\mathrm{V}(F(1,57)=5.62, r=0.30, p<0.001)$ and in $\mathrm{L}_{\mathrm{H}}$ $(F(1,57)=4.78, r=0.28, p<0.001)$. See Figure 2.b for the interaction between Modality and Group for RT.

\section{Recognition Accuracy}

Participants' RA was 1 if they responded correctly to a cue (pressed the button on the steering wheel corresponding to the appropriate urgency level) and 0 otherwise. The resulting values of RA for Modalities were as follows: $\mathrm{T}$ : 85\%, AT: $87 \%$, V: 88\%, A: 89\%, TV: 92\%, ATV: 93\%, AV: $94 \%$. Data for RA were treated as dichotomous and analysed with Cochran's Q tests. These revealed that modality $\mathrm{T}$ was less accurate than $\operatorname{AV}(Q(1)=9.14, p<$ $0.01)$, TV $(Q(1)=5.83, p<0.05)$ and $\operatorname{ATV}(Q(1)=8.33, p$ $<0.01)$, and that AT was less accurate than AV $(Q(1)=$ $4.17, p<0.05)$. The resulting values of RA for LDU were as follows: $\mathrm{L}_{\mathrm{H}}: 95 \%, \mathrm{~L}_{\mathrm{M}}: 89 \%, \mathrm{~L}_{\mathrm{L}}: 85 \%$. Cochran's $\mathrm{Q}$ tests revealed that $\mathrm{L}_{\mathrm{L}}$ was less accurate than $\mathrm{L}_{\mathrm{H}}(Q(1)=23.21, p$ $<0.001)$ and $\mathrm{L}_{\mathrm{M}}$ was less accurate than $\mathrm{L}_{\mathrm{H}}(Q(1)=12.52, p$ $<0.001)$. The resulting values of RA for Group were as follows: Control: 87\%, ASD: 93\%. Cochran's Q tests revealed that Control group was less accurate than ASD group $(Q(1)=11.80, p<0.01)$. See Figure 2.c for RA values across groups. Finally, Mann-Whitney tests for both experiments showed no significant differences in responses between Control and ASD groups in any of the factors of the DALI questionnaire.

\section{DISCUSSION}

The results showed similarities and differences between the autism spectrum and typically developed groups. Perceived urgency was similar between the groups and matched what has previously been reported using this set of warnings [22]. Differences between groups included that perceived annoyance was lower for the autism spectrum group and that the autism spectrum group was more accurate at recognizing the level of urgency and also had quicker responses when the warning included the visual modality, particularly for the vision-only warning. Consistent with claims that individuals on the autism spectrum perform differently on multisensory tasks, there was not a clear benefit of multisensory processing for the time to recognize a warning. The quickest reaction to vision-only warnings is consistent with a strong reliance on visual information in the autism spectrum group. As a guideline, visual warnings for drivers on the autistic spectrum is a viable means to communicate information quickly, as soon as they do not become an obstruction for the main driving task.

A previous study using these same warning signals with a typical population showed that ratings of urgency increased with designed urgency and that there was a pattern for both urgency and annoyance to increase as more cues were used [22]. Results from the present study broadly matched these previous results, showing that the perceived urgency increased with designed urgency and that these urgent warnings were also more annoying. A major difference, however, was that the autism spectrum group reported lower annoyance overall. This result is somewhat unexpected given that anxiety is a comorbid trait of autism. Anxiety has been theorised to potentially interfere with driving $[7,8]$ and been used to explain a trend for heart rate increase with drivers on the autism spectrum in a simulated driving experiment [24]. With higher states of anxiety as well as findings of increased sensory sensitivities for individuals higher on the autism spectrum [26], it would have seemed likely that the participants on the autism spectrum would have found the warnings more annoying. However, it has also been reported that individuals on the autism spectrum can be hypo-sensitive to sensory stimuli $[25,26]$ and hypo-sensitivity is a criterion used in diagnosing autism [1]. Thus, the pattern of low annoyance for all warnings might reflect this characteristic of autism. This is an encouraging result, since warnings that annoy drivers are less effective. Lower annoyance can therefore lead to a less demanding interaction for participants on the autistic spectrum, improving the effectiveness of warnings.

The pattern of results for both autism spectrum and typical participants for the time taken to recognize the urgency of a warning was similar to those reported previously by [22]. Warnings that were more urgent or annoying produced quicker and more precise responses. The results of recognition accuracy were also similar to this previous report in showing that recognition was greatest for high levels of urgency, less accurate in the tactile domain and most accurate for the ATV modality. However, the autism spectrum group did show overall greater recognition accuracy than the typical group. Given that both groups achieved around $90 \%$ correct responses, and in the absence of any strong interactions with sensory levels, it is difficult to give this finding a strong interpretation. Potentially the autism spectrum group performed the task more diligently or were better able to operationalize the instructions. Given the increased accuracy it would be tempting to predict that the autism spectrum group was trading off speed for accuracy, but as noted the autism spectrum group also 
demonstrated the fastest performance on the recognizing the warnings. It is therefore reasonable to assume that drivers with autism can match or even outperform typically developed ones in this recognition task, which could be useful in a demanding driving scenario, where the correct interpretation of a critical cue is essential.

A remarkable finding was that the autism spectrum group responded quicker and more accurately for warnings that included a visual modality, as vision-only was the fastest condition. The limited studies on driving in autism have reported that individuals with autism spectrum disorders perform worse than typically developed individuals. For example, the study of Sheppard and colleagues [28] found slower reaction time for reporting road hazards. They reasoned that the autism spectrum group had slower responses to hazards due to the complexity of judging the situation [9] or had difficulties in planning and producing movements to respond [12]. However, the quicker and more accurate responses of the autism spectrum group are consistent with the idea of enhanced perceptual functioning [19], meaning that for some perceptual activities, individuals on the autism spectrum will perform better than typical individuals.

The current experiment contrasted the performance of ten participants in each group. It is useful to consider how this influences the interpretation of the data. On the one hand ten participants is not a large number and it raises questions about whether the results would generalise to the entire population of drivers on the autism spectrum. However, one aspect of sensory processing in autism is that since autism is a spectrum disorder there is typically great heterogeneity of capabilities, so we would expect there to be a diversity of sensory sensitivities presented across the entire population [26]. Accordingly, a study using a large number of participants might wash out any effect. Further investigation is needed to see what aspects of performance generalize and which might be tied to particular sensory modes for particular individuals. As more is known it could be useful to characterize the profiles of individual drivers so as to obtain personally optimal warning signals.

A final consideration of the results is to note that in the present experiments, even when using the simulator, the driving task was not demanding. Future studies could examine more demanding driving situations, using more traffic or a richer driving terrain. Given that in many situations vision is overloaded with requirements of monitoring the external world and the state of the vehicle it is interesting to speculate whether the advantage seen in the autism spectrum population with rapid recognition of the warning will still obtain. Certainly, examination of the opinions of parents of teenagers on the autism spectrum regarding their son or daughters capabilities indicates that concerns are great for performance in complex situations [7]. Finally, the results of [24] showed that young drivers on the autism spectrum looked further into the distance and it was suggested that this was done to avoid the complexity of the near environment. From this one could argue that visual warnings of near events would be effective for the autism spectrum population since although they are likely scanning a simpler part of the visual scene they can still respond more quickly than typical individuals in this particular task. Finally, a possible opportunity for future studies would be to use clinical diagnosis rather than AQ score as a criterion to select participants and to obtain a larger group of ASD participants. However, given the clarity of the observed differences in the present setting, we would expect them to still stand in such a case.

\section{CONCLUSIONS}

This research contrasted how typical participants and those on the autism spectrum responded to a set of multimodal combinations of Audio, Video and Tactile modalities to alert drivers to events of varying urgency. Autism was chosen due to the growing literature indicating differences in multisensory perception therein. The results showed both common and divergent performance between the two groups. All participants appeared sensitive to the different levels of urgency encoded in the warnings as indicated by results of ratings of perceived urgency as well as speed and accuracy of response. However, a group difference was found in that the autism spectrum participants reported the warning signals to be less annoying than the typical participants. Another group difference was that the autism spectrum group showed an advantage in response time when the warning included a visual modality. In particular, recognition time by the autism spectrum group was fastest for the vision-only condition. These results highlight that while there are similarities, substantial performance differences exist between typical and autism spectrum individuals and this has implications for the design of warning signals across the entire population.

\section{REFERENCES}

1. American Psychiatric Association.Diagnostic and statistical manual of mental disorders (5th ed.). 2013.

2. Bebko, J.M., Weiss, J.A., Demark, J.L., and Gomez, P.Discrimination of temporal synchrony in intermodal events by children with autism and children with developmental disabilities without autism. Journal of Child Psychology and Psychiatry and Allied Disciplines 47, 1 (2006), 88-98.

3. Bonnel, A., Mottron, L., Peretz, I., Trudel, M., Gallun, E., and Bonnel, A.-M.Enhanced pitch sensitivity in individuals with autism: a signal detection analysis. 2003.

4. Cao, Y., van der Sluis, F., Theune, M., op den Akker, R., and Nijholt, A.Evaluating informative auditory and tactile cues for in-vehicle information systems. Proceedings of the 2nd International Conference on Automotive User Interfaces and Interactive Vehicular Applications - AutomotiveUI '10, AutomotiveUI (2010), 102. 
5. Centers for Disease Control and Prevention.Prevalence of autism spectrum disorder among children aged 8 years - autism and developmental disabilities monitoring network, 11 sites, United States, 2010. Morbidity and Mortality Weekly Report: Surveillance Summaries 63, 2 (2014), 1-21.

6. Classen, S., Monahan, M., Brown, K.E., and Hernandez, S.Driving indicators in teens with attention deficit hyperactivity and/or autism spectrum disorder. Canadian journal of occupational therapy. Revue canadienne d'ergotherapie 80, 5 (2013), 274-283.

7. Cox, N.B., Reeve, R.E., Cox, S.M., and Cox, D.J.Brief report: Driving and young adults with ASD: Parents' experiences. Journal of Autism and Developmental Disorders 42, 10 (2012), 2257-2262.

8. Daly, B.P., Nicholls, E.G., Patrick, K.E., Brinckman, D.D., and Schultheis, M.T.Driving Behaviors in Adults with Autism Spectrum Disorders. Journal of Autism and Developmental Disorders, (2014), 3119-3128.

9. Endsley, M.R.THEORETICAL UNDERPINNINGS OF SITUATION AWARENESS : A CRITICAL REVIEW Process More Data $\neq$ More Information. Most 301, 3 (2000), 3-32.

10. Ernst, M.O.Multisensory Integration: A Late Bloomer. Current Biology 18, 2008.

11. Foss-Feig, J.H., Kwakye, L.D., Cascio, C.J., et al.An extended multisensory temporal binding window in autism spectrum disorders. Experimental Brain Research 203, 2 (2010), 381-389.

12. Glazebrook, C.M., Elliott, D., and Szatmari, P.How do individuals with autism plan their movements? Journal of Autism and Developmental Disorders 38, 1 (2008), 114-126.

13. Ho, C., Reed, N., and Spence, C.Multisensory in-car warning signals for collision avoidance. Human factors 49, 6 (2007), 1107-1114.

14. Huang, P., Kao, T., Curry, A.E., and Durbin, D.R.Factors Associated With Driving in Teens With Autism Spectrum Disorders. Journal of Developmental \& Behavioral Pediatrics 33, 2012, 70-74.

15. Iarocci, G. and McDonald, J.Sensory integration and the perceptual experience of persons with autism. Journal of Autism and Developmental Disorders 36, 1 (2006), 77-90.

16. Kwakye, L.D., Foss-Feig, J.H., Cascio, C.J., Stone, W.L., and Wallace, M.T.Altered auditory and multisensory temporal processing in autism spectrum disorders. Frontiers in integrative neuroscience 4, (2011), 129.

17. Marshall, D.C., Lee, J.D., and Austria, R.A.Alerts for in-vehicle information systems: annoyance, urgency, and appropriateness. Human factors 49, 1 (2007), 145157.

18. Mongillo, E.A., Irwin, J.R., Whalen, D.H., Klaiman, C., Carter, A.S., and Schultz, R.T.Audiovisual processing in children with and without autism spectrum disorders. Journal of Autism and Developmental Disorders 38, 7 (2008), 1349-1358.

19. Mottron, L., Dawson, M., Soulières, I., Hubert, B., and Burack, J.Enhanced perceptual functioning in autism: An update, and eight principles of autistic perception. Journal of Autism and Developmental Disorders 36, 2006, 27-43.

20. Pauzie, A.A method to assess the driver mental workload: The driving activity load index (DALI). IET Intelligent Transport Systems 2, 4 (2008), 315-322.

21. Politis, I., Brewster, S. a., and Pollick, F.Evaluating multimodal driver displays under varying situational urgency. Chi 2014, (2014), 4067 - 4076.

22. Politis, I., Brewster, S., and Pollick, F.Evaluating Multimodal Driver Displays of Varying Urgency. Automotive UI 2013, ACM Press (2013), 92 - 99.

23. Politis, I., Brewster, S., and Pollick, F.Speech Tactons Improve Speech Warnings for Drivers. Automotive UI 2014, ACM Press (2014), 1-8.

24. Reimer, B., Fried, R., Mehler, B., et al.Brief Report: Examining driving behavior in young adults with high functioning autism spectrum disorders: A pilot study using a driving simulation paradigm. Journal of Autism and Developmental Disorders 43, 9 (2013), 22112217.

25. Robertson, A. and Simmons, D.R.The sensory experiences of adults with autism spectrum disorder: A qualitative analysis. Perception in press, (2015).

26. Robertson, A.E. and Simmons, D.R.The relationship between sensory sensitivity and autistic traits in the general population. Journal of Autism and Developmental Disorders 43, 4 (2013), 775-784.

27. Scott, J.J. and Gray, R.A comparison of tactile, visual, and auditory warnings for rear-end collision prevention in simulated driving. Human factors 50, 2 (2008), 264 275.

28. Sheppard, E., Ropar, D., Underwood, G., and Van Loon, E.Brief report: Driving hazard perception in autism. Journal of Autism and Developmental Disorders 40, 4 (2010), 504-508.

29. Stevenson, R. a, Siemann, J.K., Woynaroski, T.G., et al.Evidence for Diminished Multisensory Integration in Autism Spectrum Disorders. Journal of autism and developmental disorders, (2014).

30. Woodbury-Smith, M.R., Robinson, J., Wheelwright, S., and Baron-Cohen, S.Screening adults for Asperger Syndrome using the AQ: A preliminary study of its diagnostic validity in clinical practice. Journal of Autism and Developmental Disorders 35, 3 (2005), 331-335. 